\title{
Alumnado 3.0
}

\author{
José Francisco DuRÁn MEDINA \\ Universidad de Castilla La Mancha (España) \\ JoseFrancisco.Duran@uclm.es \\ Ma Rita Vega BAEZA \\ Universidad Autónoma de Zacatecas (México) \\ mrvbaeza@hotmail.com
}

Recibido: $29 / 07 / 2012$

Aceptado: 16/10/2012

\begin{abstract}
Resumen
Actualmente la sociedad está viviendo el cambio de la Web 2.0 a la Web 3.0. Esta nueva forma de vida es totalmente conocida y aceptada por la mayor parte del alumnado que tendremos en los próximos años: los nativos digitales.

Resulta obvio que los docentes y las instituciones educativas no podemos (ni debemos) quedarnos al margen de estos cambios si no queremos que la Educación, tal y como la entendemos, se quede obsoleta y pierda todo su sentido ante la vista de los educandos. Existen ya muchas y buenas experiencias al respecto, en las que los docentes empiezan a utilizar estos recursos didácticos que nos ofrece la inminente Escuela 3.0 y su alumnado responde, como no podía ser de otra manera, con buenas expectativas, gran motivación y excelentes resultados.
\end{abstract}

Palabras clave: Escuela 3.0, TIC, metodología, innovación.

\begin{abstract}
Currently the society is experiencing the change of Web 2.0 to Web 3.0. This new way of life is completely known and accepted by most of the students that we will have in the coming years: the digital natives. It is obvious that teachers and educational institutions can not (and should not) remain on the sidelines of these changes if we don't want education, as we understand it, will be obsolete and it lose all meaning in the sight of learners. There are already many good experiences in this regard, in that teachers start using these teaching resources offered by the School 3.0 imminent and students respond, like couldn't be otherwise, with high expectations, highly motivated and excellent results.
\end{abstract}

Student 3.0

Keywords: School 3.0, TIC, methodology, innovation.

\section{Referencia normalizada}

DURÁN MEDINA, José Francisco y VEGA BAEZA, Ma Rita (2012): “Alumnado 3.0”. Estudios sobre el mensaje periodístico. Vol. 18, núm. especial octubre, págs.: 293-302. Madrid, Servicio de Publicaciones de la Universidad Complutense.

Sumario: 1. Introducción. 2. Redes Sociales; 2.1. Twitter; 2.2. Facebook; 2.3. Tuenti; 2.4. Otros. 3. Redes Sociales Profesionales; 3.1. DIM Madrid; 3.2. DIM UAB: Didáctica y Multimedia; 3.3. Internet en el Aula; 3.4. Redalue; 3.5. Red EducaPR; 3.6. Rede de Estilos de Aprendizagem e EAD; 3.7. Reuna; 3.8. RUTE (Red Universitaria de Tecnología Educativa). 4. Conclusiones. 5. Referencias bibliográfícas.

\section{Introducción}

Hace ya más de quince años, los pioneros en el uso de las redes telemáticas en la escuela en nuestro país, decían que la comunicación electrónica, la apertura de la escuela al exterior gracias al correo electrónico y a las redes de telecomunicaciones existentes, representa una auténtica revolución para una escuela que hasta hace poco se encontraba cerrada dentro de las cuatro paredes y con muy poca comunicación hacia fuera (Durán, 2005). 
Sólo ha pasado algo más de una década y la frase ya está obsoleta. Actualmente la sociedad está viviendo el cambio de la Web 2.0 a la Web 3.0, la llamada Web semántica, donde cobrarán especial importancia las redes sociales y, sobre todo, la apertura a otros dispositivos además de los ordenadores.

Esta nueva forma de vida es totalmente conocida y aceptada por la mayor parte del alumnado que tendremos en los próximos años: los nativos digitales. Nuestros futuros discentes han nacido, se han criado, han aprendido a hablar, a caminar, a comunicarse utilizando estas nuevas y poderosas herramientas.

Resulta obvio que los docentes y las instituciones educativas no podemos (ni debemos) quedarnos al margen de estos cambios si no queremos que la Educación, tal y como la entendemos, se quede obsoleta y pierda todo su sentido ante la vista de los educandos. Tenemos la obligación ética y moral de renovarnos, formarnos y convertirnos (con esfuerzo y dedicación) en, al menos, inmigrantes digitales, pudiendo hacer el uso didáctico que los nuevos y numerosos materiales 3.0 nos ofrecen.

Y no sólo los docentes deben actualizarse para no quedar obsoletos. Toda la sociedad se ve afectada por este vertiginoso cambio. Sirva como ejemplo lo que bien expone Mercedes Zamarra en cuanto al periodismo, cuyo auge (periodismo digital) está directamente relacionado con el desarrollo de las nuevas tecnologías en el ámbito del ejercicio profesional y este proceso está revolucionando el oficio de periodista y, muy especialmente, los soportes y herramientas técnicas de las que éste se sirve (Zamarra, 2008).

En lo que a docencia se refiere, existen ya muchas y buenas experiencias al respecto, en las que los docentes empiezan a utilizar estos recursos didácticos que nos ofrece la inminente Escuela 3.0 y su alumnado responde, como no podía ser de otra manera, con buenas expectativas, gran motivación y excelentes resultados.

En este apartado se pretende mostrar algunas de estas experiencias realizadas en distintos centros educativos, con alumnado variado, utilizando distintas herramientas 3.0, haciendo especial hincapié en las redes sociales.

Twitter, Facebook, Tuenti, Hi5, Linkdlin... Repasaremos las funciones de algunas de estas redes que sirvan como ejemplo y mostraremos utilizaciones educativas concretas de las mismas. Pero también haremos una visita a otro tipo de redes, más profesionales, que también tienen gran interés educativo y potencian actividades grupales y trabajo colaborativo entre profesorado y alumnado, tanto a nivel nacional como internacional.

El objetivo principal que se persigue es, sin lugar a dudas, concienciar al profesorado de la necesidad de su formación en las Tecnologías de la Información y de la Comunicación (TIC), de su reciclaje continuo, de su adaptación a la sociedad actual mediante un necesario cambio metodológico que debemos llevar a cabo otorgando aún más protagonismo al alumnado en su propio proceso de Enseñanza-Aprendizaje, utilizando recursos que potencien la motivación y fomenten las relaciones personales, tanto en el sentido profesor-alumno, como profesor-profesor y alumno-alumno.

\section{Redes Sociales}

Las redes sociales son una nueva forma de comunicación frecuentemente utilizada por la mayoría de jóvenes del mundo. Se han convertido en un recurso con una gran 
fuerza de motivación en alumnos y alumnas de todas las edades que la Educación no puede, ni debe, desaprovechar.

Ya hay muchas experiencias prácticas que corroboran los beneficios de estas nuevas herramientas en el campo de la Educación.

Por ello, aunque sea de forma breve, creemos conveniente mencionar aquí también las redes sociales más utilizadas actualmente entre los jóvenes y, por tanto, las que deben ser frecuentadas y utilizadas por los centros educativos y otros grupos interesados.

\subsection{Twitter}

Twitter es una red social muy conocida y utilizada en la actualidad. Se podría definir como una aplicación en la web que permite a las personas registradas escribir pequeños textos (de hasta 140 caracteres) que pueden ser leídos por cualquiera que tenga acceso a su página.

Se tiene la opción de que los escritos sean públicos y cualquier persona pueda leer los tweets (entradas) o privado y que sólo puedan tener acceso los seguidores del autor. Cada usuario de twitter tiene una lista de "seguidos" (following) y de "seguidores" (followers). Los "seguidores" leerán los textos publicados por el "seguido" en sus páginas personales.

Hay numerosos personajes públicos que publican de forma continua lo que hacen en cada momento. Quizás por eso se está haciendo tan famoso entre los jóvenes y adolescentes.

Un buen ejemplo práctico de la utilización de Twitter con alumnos, en este caso universitarios, es el que lleva a cabo el Profesor Doctor Juan Manuel Núñez Colás, de la Universidad Autónoma de Madrid, quien da opción a sus alumnos/as a twittear opiniones provocando discusiones sobre los temas que piense pertinentes. La única regla, además de la obligatoria educación y respeto, es que los twitts hay que escribirlos en horario no lectivo. Y siempre, al comenzar las clases, se proyectan los twitts escritos provocando el debate y la motivación para escribir. La proyección se realiza mediante un software determinado gratuito y espectacular (twitterfountain, twitter-panel...).

Más ejemplos los tenemos en multitud de congresos, videoconferencias, chats, etc., donde las preguntas y/o dudas ya se empiezan a generar a través de twitter, 1legando a poder leerse y resolverse en el acto.

\subsection{Facebook}

Se dice que la historia de Facebook comenzó en la universidad americana de Harvard como una herramienta que permitía a los estudiantes permanecer en contacto entre ellos, intercambiarse notas sobre los cursos y organizar todo tipo de reuniones estudiantiles.

Ofrece la posibilidad de publicar y compartir fotos, importar imágenes desde un disco duro o desde la cámara de fotos y crear álbumes, añadir una descripción a las fotos y etiquetar a quienes salgan en ellas...

A través de Facebook, también se puede compartir otro tipo de información, publicar un vídeo o una noticia, tiene juegos, chat, mensajes privados, comentarios... 
Con todo ello, se convierte en un poderoso recurso de información, también educativa, haciendo constar cursos, congresos, artículos interesantes, noticias... Y no sólo de información, sino también de convocatoria, para establecer reuniones, visitas, salidas...
Algunos ejemplos de utilización educativa de Facebook:
Magisterio Toledo:
http://www.facebook.com/\#!/educaciontoledoUCLM
Universidad de Castilla La Mancha (UCLM):
http://www.facebook.com/\#!/universidadcastillalamancha

\subsection{Tuenti}

Con la ayuda de la enciclopedia Wikipedia podemos afirmar que a fecha de febrero de 2012, Tuenti tiene más de 13 millones de usuarios, obteniendo un crecimiento del $33 \%$ en el último año. El sitio está enfocado principalmente a la población española. Permite al usuario crear su propio perfil, añadir a otros usuarios como amigos e intercambiar mensajes. Además, los usuarios se pueden unir a páginas de intereses comunes, gestión de eventos, subida fotos y vídeos.

Quizás menos académico y más informal, con mayor éxito entre la juventud y adolescencia, comparte prácticamente las mismas funciones y posibilidades que Facebook. No obstante, uno de los mayores problemas de Tuenti es su propio éxito, ya que el volumen total de información hace que falle a menudo.

Algunas experiencias a nivel educativo:

Universidad de Alcalá:

http://www.tuenti.com/\#m=Page\&func=index\&page_key=1_2003_73633277

Universidad de Huelva.

www.tuenti.com/universidaddehuelva

Biblioteca de la Universidad Carlos III:

http://www.tuenti.com/\#m=Page\&func=index\&page_key=2_273522_425

\subsection{Otros}

Sin el ánimo de enumerar cada una de las redes sociales existentes ya que sería un laborioso trabajo al existir un número tan elevado de ellas, simplemente queremos observar que cualquier red social, tanto profesional como social, formal o informal, pueden suponer un valioso recurso didáctico si se utiliza con ese fin, planteando los necesarios objetivos, realizando las tareas previstas, desarrollando los temas pertinentes, valorando y evaluando su utilización, actualizándolo habitualmente...

Además de las mencionadas, podremos hacer uso de Google + , Linkdlin, MySpace, Ning, Tagged, MyYearbook, Meetup, Bebo, Multiply, Orkut, Skyrock, Badoo, StumbleUpon, Delicious, Foursquare, MyOpera, Kiwibox, Hi5...

\section{Redes Sociales Profesionales}

Reconociendo de antemano el elevado número de redes sociales educativas de calidad existentes, se intentarán nombrar algunas de ellas, con las que los autores de este capítulo se sienten comprometidos y han colaborado habitualmente con experiencias 
personales en cada una de las redes educativas que se van a exponer a continuación por orden alfabético:

3.1. DIM Madrid: www.uam.es/melchor.gomez/dim

Como ellos mismos expresan en su página de presentación, es un grupo de profesores y profesionales del mundo educativo.

Son de diferentes niveles y disciplinas que creen en el uso de las Tecnologías de la Información y la Comunicación para la mejora de la enseñanza. Comparten sus ideas, conocimientos y proyectos, buscando que la sinergia que generan beneficie a todos los integrantes.

Es uno de los grupos de trabajo del Grupo DiM. Mantienen contactos a través de una lista de correo propia y se reúnen 4 veces al año para compartir experiencias e intuiciones.

Docentes de distintas universidades y centros educativos nacionales e internacionales colaboramos en esta red, siendo el Profesor Doctor Melchor Gómez García quien lo encabeza y dirige: Univ. Autónoma de Madrid, Universidad Carlos III de Madrid, UNED, Universidad Politécnica de Madrid, Universidad de Castilla La Mancha, Universidad Pedagógica Libertador (Venezuela), Universidad del Sagrado Corazón en Sao Paulo (Brasil), Universidad Católica Andrés Bello de Caracas (Venezuela), Universidad Nacional Pedro Ruiz Gallo en Lambayeque (Perú), Universidad Nacional Mayor de San Marcos de Lima (Perú), Universidad de los Lagos (Chile), Pontificia Universidad Católica de Perú, Universidad Juan Misael Saracho de Tarija (Bolivia), IES Clara del Rey de Madrid, Colegio Arcángel Rafael de Madrid, Centro de Apoyo al Profesorado de Madrid Centro, Colegio de San Sebastián en El Boalo (Madrid), etc.

\subsection{DIM UAB. Didáctica y Multimedia: http://dim.pangea.org/}

Se autodefine como grupo de investigación y comunidad de aprendizaje de profesores, gestores educativos y especialistas de empresas que usan las TIC para mejorar enseñanza y aprendizaje; para investigar y compartir materiales, conocimiento, iniciativas, experiencias.

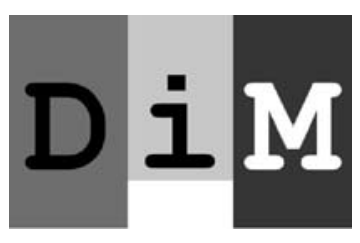

El profesor Dr. Pere Marqués, de la Universitat Autònoma de Barcelona dirige este estupendo proyecto donde podemos encontrar multitud de recursos distribuidos entre el blog, foros con infinidad de temáticas todas ellas relacionadas con la Educación, proyectos de investigación, vídeos, fotografías y otros recursos.

Participantes de todo el mundo participan (o participamos), generalmente recibiendo más de lo que damos. Pere Marqués, Eloy López Meneses, José M. Giráldez, Javier Gómez Torres, Sergio Carrera, Damiá Mendoza Zambrano, Jordi Casals, Belén Argote Marqués, Mónica Moya López, Toni Perulles Rull y así hasta llegar a los dos mil ochocientos cuarenta y cuatro miembros de la red.

\subsection{Internet en el Aula: http://internetaula.ning.com/}

Se trata de una red social docente para una educación del siglo XXI en la que 11576 miembros docentes comparten experiencia y recursos para el uso de Internet en el aula. 
Participan en su desarrollo el Instituto Nacional de Tecnologías Educativas y de Formación del Profesorado y el Ministerio de Educación, Cultura y Deporte del Gobierno de España.

\section{Internet en el Aula}

Red social docente para una educación del siglo $X X$

Entre sus apartados están Mi página, con los subapartados Mis amigos, Mi blog, Mi correo, Mis discusiones, Mis eventos, Mis grupos, Mis fotos y Mis ideas; Foro, con Proyectos, Buenas prácticas 2.0, Herramientas, Materiales didácticos, Dispositivos, Competencias, Metodologías y Noticias; Grupos, con las posibilidad de Acceso rápido a grupos, Grupos por áreas, Grupos más populares, Grupos más activos, Grupos más recientes y Ayuda sobre los grupos; Y otras muchas más: Blogs, Multimedia, BPrácticas 2.0, Talleres, Webinars, Chats, Miembros...

Entre los grupos más destacados en la actualidad se pueden encontrar Mundos virtuales, aplicaciones educativas; Matemáticas \& TIC; Trucos TIC para el aula; TAAC Herramientas portables; Competencias y Educación para el desarrollo.

Interesantes discusiones como las dedicadas a Herramientas que nuestr@s alumn@s pueden usar sin registrarse ¿Cuáles son las mejores?; Uso de la pizarra digital en el aula; Posibilidades educativas de los mundos virtuales; Terminales multipuesto con software libre en las aulas; uso de herramientas tecnológicas como mediaciones pedagógicas en el proceso Enseñanza Aprendizaje...

\subsection{Redalue: http://www.redalue.com/cms}

La Red Colaborativa de Gestión del Conocimiento para América Latina y Unión Europea está formada por un grupo de profesores entusiastas, conscientes de la necesidad de compartir conocimiento.

Su línea de acción se centra en la formación sobre seis ejes de conocimiento para lograr la adquisición de competencias en profesores y estudiantes: Tecnologías para la Educación y el Conocimiento, Estilos de aprendizaje, Inteligencia Emocional, Gestión del conocimiento, Competencias EEES y eLearning.

Han adquirido el compromiso de mejorar la calidad de las Instituciones y el aprovechamiento óptimo de los estudiantes, con la pretensión de la adquisición de competencias necesarias para una integración eficaz en el futuro profesional de los estudiantes.

Con los Profesores Doctores de la UNED Catalina M. Alonso y Domingo J. Gallego a la cabeza, los países que la integran son España, Portugal, Francia, Argentina, Bolivia, Brasil, Chile, Costa Rica, Colombia, Ecuador, El Salvador, México, Perú, U.S.A. y Venezuela.

\subsection{Red EducaPR: http://www.educapr.org/}

Con el subtítulo "Web 2.0, Tecnología y Educación", la Red de Educadores Puertorriqueños EducaPR es una red social y educativa que promueve el uso de la tecnología, Internet y Web 2.0 en la Educación en las diversas zonas de Puerto Rico, Estados Unidos, otros países de Latinoamérica y España.

Realiza conferencias libre de costo sobre temas actuales de tecnología y Educación. Las conferencias se transmiten por Internet, pudiendo participar de forma presencial y/o virtual. 
Acciones posibles: invitar a otros educadores a participar, actualizar foto y página principal, escribir en el foro o en el blog, añadir fotos de actividades educativas, añadir videos con mensajes de educación o tecnología, participar en las conferencias, seguir las novedades en las redes sociales Twitter, Facebook o Google +, compartir actividades educativas, conferencias o enlaces, unirse o crear un grupo...

Además de las habituales funciones de presentación de la página y pestaña para invitar a amigos, otras posibilidades que nos ofrece esta red son Mi página, Blogs, Foro, Fotos, Vídeos, Eventos, Grupos, Congreso Blogs EducaPR, Conferencias 2011, Conferencias 2010, Noticias de EducaPR, Podcasts, WordCampPonce, Revista EducaPR 2012, Twitter-Verano-2012...

Esta red profesional docente, con Jeanette Delgado Nazario en cabeza, está trabajando actualmente en varios Proyectos Educativos, como Edutuiteros Boricuas, Blogs Destacados de EducaPR, Comparte tu blog con EducaPR y Crear cursos en Edu2.0. También se está llevando a cabo el Proyecto Colaborativo TecnoPaz, con el lema "Unidos por la Tecnología podemos promover la Paz".

3.6. Rede de Estilos de Aprendizagem e EAD: http://sites.google.com/site/estilosead/ Como se puede deducir a través de su mismo nombre, se trata de una red cuyo lenguaje habitual (sin descartar otros también utilizados, como el español), es el portugués, lengua oficial de su autora, la Profesora Doctora de origen brasileño Daniela Melaré.

Es una red docente básicamente dedicada al tema de los Estilos de Aprendizaje, donde autores de diferentes universidades internacionales aportamos ideas y colaboramos en proyectos, congresos, artículos, libros, etc., al respecto.

En su página inicial, además de las ya típicas pestañas que nos invitan a participar en los foros, eventos, cursos, vídeos, etc., tiene la originalidad de poder realizar el Test CHAEA online (con la posibilidad de descargarlos por escrito) sobre Estilos de Aprendizaje para descubrir cuál o cuáles de estos estilos (activo, reflexivo, teórico y pragmático) son los que predominan en nuestro modus operandi.

\subsection{Reuna: http://www.reuna.cl/}

Como nos desvelan en su página de presentación, la Corporación Red Universitaria Nacional (REUNA) es una iniciativa de colaboración que, impulsada por instituciones de investigación y educación superior, cuenta con la única infraestructura tecnológica de Red Académica dedicada a la investigación, educación e innovación en Chile.

REUNA está interconectada globalmente con las Redes Académicas Internacionales. A través de esta conexión, las instituciones que la integran conectan a sus académicos con sus pares en América Latina (mediante RedCLARA), América del Norte (Internet2 y Canarie), Europa (GÉANT), Asia (APAN) y Oceanía (AARNET).

- Provee servicios avanzados de comunicación y conectividad nacional e internacional.

- Propicia la formación de comunidades de colaboración docentes y de investigación.

- Articula iniciativas y proyectos que promueven el trabajo conjunto entre los socios y de estos con sus pares internacionales.

- Impulsa la investigación, desarrollo e innovación en nuevas tecnologías. 
REUNA apoya, conecta y comunica a sus socios en el concierto global, proporcionando oportunidades estratégicas en materias de tecnologías de información y comunicación y servicios avanzados, para la colaboración e integración global de la comunidad de educación superior, ciencia e innovación nacional.

Como a esta red le gusta decir de sí misma, REUNA es Ciencia y Educación en Red.

Entre sus servicios habría que destacar la posibilidad de realizar videoconferencias entre distintas universidades internacionales, actividad que tuvimos el placer de desarrollar junto a varios grupos de alumnos/as de la Facultad de Educación de Toledo, en las que ellos y ellas expusieron a otros alumnos/as y docentes varios trabajos realizados sobre la fotografía y el vídeo educativos. Todas estas videoconferencias quedan luego expuestas en la interesante videoteca de su página para quienes quieran verlas o descargárselas.

De igual modo, se realizan talleres, proyectos de investigación internacionales, asistencia a eventos...

3.8. RUTE (Red Universitaria de Tecnología Educativa): http://www.rute.edu.es/ Finalmente, la Red Universitaria de Tecnología Educativa (RUTE), una asociación académica sin ánimo de lucro constituida por docentes e investigadores universitarios interesada en promover las aplicaciones educativas de las tecnologías de la información y Comunicación.

Esta asociación nació en las XIV JUTE (Jornadas Universitarias de Tec-

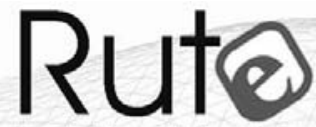

Red Universitaria de Tecnología Educativa nología Educativa), celebradas en Santiago de Compostela, junio de 2006.

Tiene integrada en su página inicial la Red Docente de Tecnología Educativa, red o comunidad social de docentes e investigadores latinoamericanos preocupados por el uso pedagógico de las TIC (Tecnologías de la Información y Comunicación).

Es una red destinada a los docentes universitario o formadores de profesorado de cualquier nivel educativo que desean compartir su experiencia, debatir sobre un tema relacionado con esta temática, crear un blog, participar en un grupo de trabajo, aportar fotos, vídeos o cualquier otro material que considere de interés.

\section{Conclusiones}

Hemos dado un breve pero cálido paseo por este frondoso bosque de las redes sociales atendiendo únicamente a sus posibilidades educativas.

Antes de concluir queremos advertir que las posibilidades que nos ofrecen estos recursos, así como cualquiera si cae en manos de lo que entendemos por un buen profesor, pueden llegar a ser infinitas. Acabarán donde termine la imaginación y creatividad del docente.

Además de las redes expuestas existen otras muchas, tanto a nivel profesional como exclusivamente social. Cada docente debe valorar cuál o cuáles pueden ser útiles para su propósito, sus objetivos, teniendo en cuenta su situación particular y la de sus discentes. 
Lo que queda claro es que los docentes no podemos quedarnos quietos, mirando. Hay que coger este tren, nunca mejor llamado AVE dada su rapidez, su cambio continuo.

Sirva como claro ejemplo de lo expuesto la Alianza Estratégica entre Redes Internacionales de Educación de la cual forman parte ambos autores de este documento. Esta alianza, compuesta por diversos profesores con intención de aunar esfuerzos en pro de la investigación educativa y organización de eventos conjuntos, está formada hasta el momento por la Red Iberoamericana de Educación Superior, Humanidades y Nuevas Tecnologías (cuyos coordinadores son José Francisco Durán Medina y Ma Rita Vega Baeza) y la Red Internacional, Interinstitucional e Interdisciplinar de Investigación Educativa "Políticas, Cultura y Diversidad Educativa". Entre sus miembros destacan integrantes de los Cuerpos Académicos "Estudios de filosofía, arte y literatura" y "Políticas Educativas", ambos pertenecientes al Programa de Maestría en Investigaciones Humanísticas y Educativas de la Unidad Académica de Docencia Superior de la Universidad Autónoma de Zacatecas (México), del Grupo de Investigación de la Facultad de Educación de Toledo (Universidad de Castilla La Mancha España), del Cuerpo Académico de la Universidad de Victoria (Canadá), del Grupo de Investigación "Educación de Adultos" de la Cátedra de Andragogía del Instituto de la Educación Pedagógica y Educación de Adultos de la Academia Nacional de Ciencias Pedagógicas de Ucrania, del Grupo de Investigadores de la Universidad de Greifswald y del Instituto de Karlsruhe (Alemania).
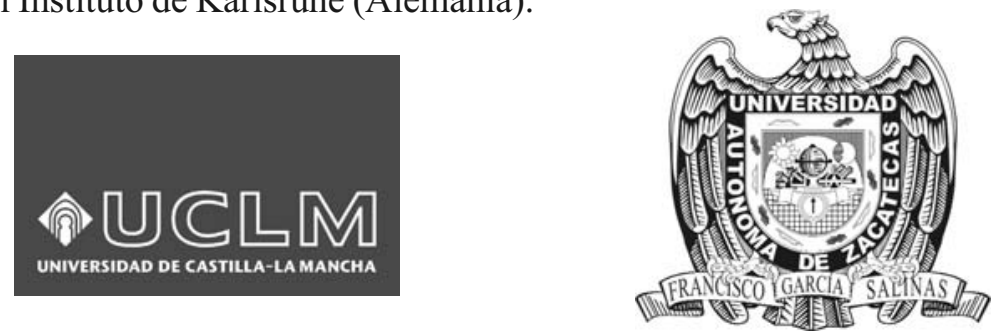

Se trata de un Grupo de compañeros/as deseosos/as de recibir colaboradores/as para seguir aumentando el número y la calidad de sus producciones.

Y aún no hemos hablado de otras redes, más privadas, con idénticas posibilidades, que invitamos a visitar, conocer, crear, trabajar. Llamémoslas Moodle, Joomla, cualquier web, blog, wiki... Pero eso..., quizás en otra ocasión.

\section{Referencias bibliográfícas}

DIM UAB. Grupo de Investigación Didáctica y Multimedia Barcelona. Disponible en: http://dim.pangea.org/. Consultado el 1 de julio de 2012.

DIM Madrid: Grupo de Investigación Didáctica y Multimedia Madrid. Disponible en: http://www.uam.es/melchor.gomez/dim/. Consultado el 1 de julio de 2012.

DURÁN MEDINA, José Francisco (2005): "Herramientas educativas en internet". Revista de Comunicación SEECI, $\mathrm{n}^{\circ} 12$, Noviembre 2005. Madrid. Universidad Complutense, recuperado el 1 de noviembre de 2012, en la siguiente dirección: http://www.ucm.es/info/seeci/Numeros/Numero\%2012/DATOS.html 
Internet en el Aula. Red Social Docente. Disponible en: http://internetaula.ning.com/. Consultado el 1 de julio de 2012.

Red EducaPR. Red Social Educativa. Disponible en: http://www.educapr.org/. Consultado el 1 de julio de 2012.

Redalue. Red Colaborativa de Gestión del Conocimiento. Disponible en: http://www.redalue.com/cms/. Consultado el 1 de julio de 2012.

Rede de Estilos de Aprendizagem e EAD. Red de Estilos de Aprendizaje. Disponible en: http://sites.google.com/site/estilosead/. Consultado el 1 de julio de 2012.

Reuna. Red Académica Internacional. Disponible en: http://www.reuna.cl/. Consultado el 1 de julio de 2012.

RUTE. Red Universitaria de Tecnología Educativa. Disponible en: http://www.rute.edu.es/. Consultado el 1 de julio de 2012.

Wikipedia. Enciclopedia Libre. Disponible en: http://es.wikipedia.org/. Consultado el 1 de julio de 2012.

ZAMARRALópez, $\mathrm{M}^{\mathrm{a}}$ de las Mercedes (2008): "El periodismo digital y la nueva formación cíber-periodística". Revista de Comunicación SEECI, $\mathrm{n}^{\circ}$ 15, Marzo 2008. Madrid. Universidad Complutense, recuperado el 1 de noviembre de 2012, en: http://www.ucm.es/info/seeci/Numeros/Numero 15/DATOS.html

\section{José Francisco DURÁN MEDINA \\ $M^{a}$ Rita VEGA BAEZA}

Correo electrónico (e-mail) del autor/es:

JoseFrancisco.Duran@uclm.es

mrvbaeza@hotmail.com

Dirección postal:

Despacho 1.28. Edificio Sabatini.

Facultad de Educación de Toledo.

Universidad de Castilla La Mancha (UCLM)

45071 Toledo (España).

Filiación institucional:

Universidad de Castilla La Mancha (España).

Universidad Autónoma de Zacatecas (México). 